\title{
Computational Methods for Three Coupled Nonlinear Schrödinger Equations
}

\author{
M. S. Ismail, S. H. Alaseri \\ Department of Math, King Abdulaziz University, Jeddah, Kingdom of Saudi Arabia \\ Email: msismail@kau.edu.sa
}

How to cite this paper: Ismail, M.S. and Alaseri, S.H. (2016) Computational Methods for Three Coupled Nonlinear Schrödinger Equations. Applied Mathematics, 7, 21102131.

http://dx.doi.org/10.4236/am.2016.717168

Received: September 8, 2016

Accepted: November 12, 2016

Published: November 15, 2016

Copyright (c) 2016 by authors and Scientific Research Publishing Inc. This work is licensed under the Creative Commons Attribution International License (CC BY 4.0).

http://creativecommons.org/licenses/by/4.0/ (c) (7) Open Access

\begin{abstract}
In this work, we will derive numerical schemes for solving 3-coupled nonlinear Schrödinger equations using finite difference method and time splitting method combined with finite difference method. The resulting schemes are highly accurate, unconditionally stable. We use the exact single soliton solution and the conserved quantities to check the accuracy and the efficiency of the proposed schemes. Also, we use these methods to study the interaction dynamics of two solitons. It is found that both elastic and inelastic collision can take place under suitable parametric conditions. We have noticed that the inelastic collision of single solitons occurs in two different manners: enhancement or suppression of the amplitude.
\end{abstract}

\section{Keywords}

Three Coupled Nonlinear Schrodinger Equations, Finite Difference Method, Time Splitting Method, Interaction of Solitons

\section{Introduction}

In recent years, the concept of soliton has been receiving considerable attention in optical communications, since soliton is capable of propagating over long distances without change of shape and velocity. It has been found that the soliton propagating through optical fiber arrays is governed by a set of equations related to the coupled nonlinear Schrödinger equation [1] [2].

$$
i q_{j t}+q_{j x x}+2\left[\sum_{p=1}^{N}\left|q_{p}\right|^{2}\right] q_{j}=0, j=1,2, \cdots, N,
$$

where $i^{2}=-1, q_{j}$ is the envelope or the amplitude of the jth wave packets. Equation (1) reduces to the standard nonlinear Schrödinger equation for $N=1$, to Manakov integrable system for $N=2$, and recently for this case the exact two soliton solution 
obtained and novel shape changing in elastic collision property has been brought out. The system for $N=3$ is of physical interest, in optical communication, and in biophysics this system can be used to study the lunching and propagation of solitons along the three spines of an alpha-helix shape changing in protein [1] [2] [3] [4]. In this work, we are going to derive some numerical methods for solving the three coupled nonlinear Schrödinger equations (3-CNLS)

$$
\begin{aligned}
& i q_{1 t}+q_{1 x x}+2\left(\left|q_{1}\right|^{2}+\left|q_{2}\right|^{2}+\left|q_{3}\right|^{2}\right) q_{1}=0, \\
& i q_{2 t}+q_{2 x x}+2\left(\left|q_{1}\right|^{2}+\left|q_{2}\right|^{2}+\left|q_{3}\right|^{2}\right) q_{2}=0, \\
& i q_{3 t}+q_{3 x x}+2\left(\left|q_{1}\right|^{2}+\left|q_{2}\right|^{2}+\left|q_{3}\right|^{2}\right) q_{3}=0,
\end{aligned}
$$

with initial conditions

$$
q_{j}(x, 0)=g_{j}(x), x_{L} \leq x \leq x_{R}, \quad j=1,2,3,
$$

and the homogenous boundary conditions

$$
q_{j}\left(x_{L}, t\right)=q_{j}\left(x_{R}, t\right)=0, j=1,2,3,
$$

The exact solution of the 3-coupled nonlinear Schrödinger equation [1] [2] is given by

$$
\begin{gathered}
q_{j}(x, t)=A_{j} k_{1 R} \mathrm{e}^{i \lambda_{I}} \operatorname{sech}\left(\lambda_{R}+\frac{R}{2}\right), \\
A_{j}=\frac{\alpha_{j}}{\Delta}, \Delta^{2}=\sum_{j=1}^{3}\left|\alpha_{j}\right|^{2}, \mathrm{e}^{R}=\frac{\Delta^{2}}{\left(k_{1}+k_{1}^{*}\right)^{2}}, \lambda=k_{1}\left(x+i k_{1} t\right), j=1,2,3,
\end{gathered}
$$

where $\alpha_{1}^{(j)}, k_{1}, j=1,2,3$, are four arbitrary complex parameters. Further $k_{1 R} A_{j}$ gives the amplitude of the $j$ th mode and $2 k_{1 I}$ the soliton velocity.

Many numerical methods for solving the coupled nonlinear Schrödinger equation are derived in the last two decades. Finite difference and finite element methods are used to solve this system by Ismail [5]-[10]. A conservative compact finite difference schemes are given in [11] [12]. In [3] [4], Xing Lü studied the bright soliton collisions with shape change by intensity for the coupled Sasa-Satsuma system in the optical fiber communications. A higher order exponential time differencing scheme for system of coupled nonlinear Schrödinger equation is given in [13]. A semi-explicit multi-sypmlectic splitting scheme for 3-coupled nonlinear Schrödinger equation is given in [14]. Many researchers are used time splitting method for solving the coupled non-linear schrödinger equation, the basic idea in this method is to split the original system into a linear subsystem and nonlinear subsystem, The splitting simplify the problem, since the linear problem is uncoupling, relatively easy to solve and the nonlinear problem can be solved exactly due to their point-wise conservation law, for more details see [15]-[23].

To avoid complex computations we assume

$$
q_{1}=u_{1}+i u_{2}, q_{2}=u_{3}+i u_{4}, q_{3}=u_{5}+i u_{6}
$$

where $u_{i}(x, t), i=1,2, \cdots, 6$ are real functions, the systems (2)-(4) can be written as 


$$
\begin{array}{ll}
u_{1 t}+u_{2 x x}+\omega u_{2}=0, & u_{2 t}-u_{1 x x}-\omega u_{1}=0, \\
u_{3 t}+u_{4 x x}+\omega u_{4}=0, & u_{4 t}-u_{3 x x}-\omega u_{3}=0, \\
u_{5 t}+u_{6 x x}+\omega u_{6}=0, & u_{6 t}-u_{5 x x}-\omega u_{5}=0,
\end{array}
$$

where

$$
\omega=\left(u_{1}^{2}+u_{2}^{2}\right)+\left(u_{3}^{2}+u_{4}^{2}\right)+\left(u_{5}^{2}+u_{6}^{2}\right)
$$

The resulting systems (8)-(10) can be written in a matrix-vector form as

$$
\frac{\partial \boldsymbol{v}_{j}}{\partial t}+A \frac{\partial^{2} \boldsymbol{v}_{j}}{\partial x^{2}}+A \omega \boldsymbol{v}_{j}=\mathbf{0}, \quad j=1,2,3
$$

where

$$
\boldsymbol{v}_{1}=\left[\begin{array}{l}
u_{1} \\
u_{2}
\end{array}\right], \boldsymbol{v}_{2}=\left[\begin{array}{l}
u_{3} \\
u_{4}
\end{array}\right], \boldsymbol{v}_{3}=\left[\begin{array}{l}
u_{5} \\
u_{6}
\end{array}\right], A=\left[\begin{array}{cc}
0 & 1 \\
-1 & 0
\end{array}\right],
$$

Proposition 1 The three coupled nonlinear Schöridinger equations have the conserved quantities

$$
\begin{gathered}
I_{1}=\int_{x_{L}}^{x_{R}}\left|q_{1}(x, t)\right|^{2} \mathrm{~d} x=\cdots=\int_{x_{L}}^{x_{R}}\left|q_{1}(x, 0)\right|^{2} \mathrm{~d} x \\
I_{2}=\int_{x_{L}}^{x_{R}}\left|q_{2}(x, t)\right|^{2} \mathrm{~d} x=\cdots=\int_{x_{L}}^{x_{R}}\left|q_{2}(x, 0)\right|^{2} \mathrm{~d} x \\
I_{3}=\int_{x_{L}}^{x_{R}}\left|q_{3}(x, t)\right|^{2} \mathrm{~d} x=\cdots=\int_{x_{L}}^{x_{R}}\left|q_{3}(x, 0)\right|^{2} \mathrm{~d} x
\end{gathered}
$$

Proof : we consider the first conserved quantity (13), from (8) we have

$$
\begin{gathered}
\frac{\partial u_{1}(x, t)}{\partial t}+\frac{\partial^{2} u_{2}(x, t)}{\partial x^{2}}+\omega u_{2}(x, t)=0, \\
\frac{\partial u_{2}(x, t)}{\partial t}-\frac{\partial^{2} u_{1}(x, t)}{\partial x^{2}}-\omega u_{1}(x, t)=0,
\end{gathered}
$$

by multiplying (16) by $u_{1}(x, t)$ and (17) by $u_{2}(x, t)$ and by adding the resulting equations to obtain

$$
\frac{\partial}{\partial t}\left[u_{1}^{2}(x, t)+u_{2}^{2}(x, t)\right]+\frac{\partial}{\partial x}\left[u_{1}(x, t) \frac{\partial u_{2}(x, t)}{\partial x}-u_{2}(x, t) \frac{\partial u_{1}(x, t)}{\partial x}\right]=0 .
$$

By integrating Equation (18) with respect to $x$ from $x_{L}$ to $x_{R}$ and using the vanishing boundary conditions to obtain

$$
\frac{\partial}{\partial t} \int_{x_{L}}^{x_{R}}\left[u_{1}^{2}(x, t)+u_{2}^{2}(x, t)\right] \mathrm{d} x=0,
$$

and this is the proof of the first conserved quantity (13). The other two conserved quantities (14) and (15) can be proved in the same way.

The exact values of the conserved quantities using the exact soliton solution (6) are given by the following formula

$$
I_{j}=\frac{2 k_{1 R}\left|\alpha_{j}\right|^{2}}{\left[\left|\alpha_{1}\right|^{2}+\left|\alpha_{2}\right|^{2}+\left|\alpha_{3}\right|^{2}\right]}, j=1,2,3 .
$$


The paper is organized as follows. In Section 2, we derived a second order CrankNicolson scheme for solving the proposed system. The fourth order compact difference scheme is derived in Section 3. In Section 4, we present two fixed point schemes to solve the block nonlinear tridiagonal systems obtained in Sections 2 and 3. To avoid the nonlinearity obtained in the previous sections, we present time splitting method to solve the 3-CNLS in Section 5. The numerical comparison of the derived methods are reported in Section 6. Finally, we draw some conclusions in Section 7.

\section{Second Order Crank-Nicolson Scheme}

In order to develop a numerical method for solving the system given in (12), the region $R=\left[x_{L}<x<x_{R}\right] \times[t>0]$ will be covered with a rectangular mesh of points with coordinates,

$$
\begin{gathered}
x_{m}=x_{L}+m h, \quad m=0,1,2, \cdots, M \\
t=t_{n}=n k, n=0,1,2, \cdots
\end{gathered}
$$

where $h$ and $k$ are the space and time increments respectively. We denote the exact and numerical solution at the grid point $\left(x_{m}, t_{n}\right)$ by $\boldsymbol{v}_{j, m}^{n}$ and $\boldsymbol{V}_{j, m}^{n+1}$, respectively. We approximate the space derivative by the second order central difference formula

$$
\frac{\partial^{2} u\left(x_{m}, t\right)}{\partial x^{2}}=\frac{1}{h^{2}}\left(u\left(x_{m-1}, t\right)-2 u\left(x_{m}, t\right)+u\left(x_{m+1}, t\right)\right)=\frac{1}{h^{2}} \delta_{x}^{2} u\left(x_{m}, t\right)+O\left(h^{2}\right),
$$

where $\delta_{x}^{2}$ is the second order central difference operator. The Crank-Nicolson scheme for the 3-CNLS equation is given by

$$
\boldsymbol{V}_{j, m}^{n+1}-\boldsymbol{V}_{j, m}^{n}+\frac{1}{2} r A \delta_{x}^{2}\left[\boldsymbol{V}_{j, m}^{n+1}+\boldsymbol{V}_{j, m}^{n}\right]+\frac{1}{4} k w_{m}^{n+1} A\left(\boldsymbol{V}_{j, m}^{n+1}+\boldsymbol{V}_{j, m}^{n}\right)=\mathbf{0},
$$

where

$$
w_{m}^{n+1}=\sum_{j=1}^{3}\left(\left|q_{j, m}^{n+1}\right|^{2}+\left|q_{j, m}^{n}\right|^{2}\right), r=\frac{k}{h^{2}}, j=1,2,3 .
$$

The scheme in (20) is of second order accuracy in both directions space and time, and it is unconditionally stable using von-Neumann stability analysis, see Ismail [8]. A nonlinear block tridiagonal system must be solved at each time step. Fixed point method is used to do this job and this will be discussed later.

\section{Fourth Order Compact Difference Scheme}

A highly accurate finite difference scheme can be obtained by using the fourth order Padè compact difference approximation for the spatial discretization

$$
\frac{\partial^{2} u\left(x_{m}, t\right)}{\partial x^{2}}=\frac{1}{h^{2}}\left(1+\frac{1}{12} \delta_{x}^{2}\right)^{-1} \delta_{x}^{2} u\left(x_{m}, t\right)+O\left(h^{4}\right)
$$

together with the Crank-Nicolson scheme, this will lead us to the compact finite difference scheme

$$
\boldsymbol{V}_{j, m}^{n+1}-\boldsymbol{V}_{j, m}^{n}+\frac{1}{2} r A\left(1+\frac{1}{12} \delta_{x}^{2}\right)^{-1} \delta_{x}^{2}\left[\boldsymbol{V}_{j, m}^{n+1}+\boldsymbol{V}_{j, m}^{n}\right]+\frac{1}{2} k w_{m}^{n+1} A\left(\boldsymbol{V}_{j, m}^{n+1}+\boldsymbol{V}_{j, m}^{n}\right)=\mathbf{0},
$$


where

$$
w_{m}^{n+1}=\sum_{j=1}^{3}\left(\left|q_{j, m}^{n+1}\right|^{2}+\left|q_{j, m}^{n}\right|^{2}\right) .
$$

The scheme (21) can be written in a block tridiagonal form as

$$
\begin{aligned}
& \frac{1}{12}\left(\boldsymbol{V}_{j, m-1}^{n+1}-\boldsymbol{V}_{j, m-1}^{n}\right)+\frac{10}{12}\left(\boldsymbol{V}_{j, m}^{n+1}-\boldsymbol{V}_{j, m}^{n}\right)+\frac{1}{12}\left(\boldsymbol{V}_{j, m+1}^{n+1}-\boldsymbol{V}_{j, m+1}^{n}\right) \\
& +\frac{1}{2} r A\left[\boldsymbol{V}_{j, m-1}^{n+1}-2 \boldsymbol{V}_{j, m}^{n+1}+\boldsymbol{V}_{j, m+1}^{n+1}\right]+\frac{1}{2} r A\left[\boldsymbol{V}_{j, m-1}^{n}-2 \boldsymbol{V}_{j, m}^{n}+\boldsymbol{V}_{j, m+1}^{n}\right] \\
& +\frac{k}{12}\left[\boldsymbol{G}_{j, m-1}^{n+1}+10 \boldsymbol{G}_{j, m}^{n+1}+\boldsymbol{G}_{j, m+1}^{n+1}\right]=\mathbf{0},
\end{aligned}
$$

where

$$
\boldsymbol{G}_{j, m}^{n+1}=\frac{1}{4} \omega_{m}^{n+1} A\left(\boldsymbol{V}_{j, m}^{n+1}+\boldsymbol{V}_{j, m}^{n}\right), j=1,2,3 .
$$

The method is of second order accuracy in time and fourth order in space, it is implicit unconditionally stable, see Ismail [9]. The resulting system is a block nonlinear tridiagonal system and can be solved by fixed point method and this will be discussed next. From the previous methods, we can derive the generalized finite difference scheme

$$
\begin{aligned}
& \sigma\left(\boldsymbol{V}_{j, m-1}^{n+1}-\boldsymbol{V}_{j, m-1}^{n}\right)+(1-2 \sigma)\left(\boldsymbol{V}_{j, m}^{n+1}-\boldsymbol{V}_{j, m}^{n}\right)+\sigma\left(\boldsymbol{V}_{j, m+1}^{n+1}-\boldsymbol{V}_{j, m+1}^{n}\right) \\
& +\frac{1}{2} r A\left[\left(\boldsymbol{V}_{j, m-1}^{n+1}+\boldsymbol{V}_{j, m-1}^{n}\right)-2\left(\boldsymbol{V}_{j, m}^{n+1}+\boldsymbol{V}_{j, m}^{n}\right)+\left(\boldsymbol{V}_{j, m+1}^{n+1}+\boldsymbol{V}_{j, m+1}^{n}\right)\right] \\
& +k\left[\sigma \boldsymbol{G}_{j, m-1}^{n}+(1-2 \sigma) \boldsymbol{G}_{j, m}^{n}+\sigma \boldsymbol{G}_{j, m+1}^{n}\right]=0,
\end{aligned}
$$

where

$$
\boldsymbol{G}_{j, m}^{n}=\frac{1}{4} \omega_{m}^{n+1} A\left(\boldsymbol{V}_{j, m}^{n+1}+\boldsymbol{V}_{j, m}^{n}\right), j=1,2,3,
$$

for arbitrary parameter $\sigma$. The scheme is second order in time and space for $\sigma \neq \frac{1}{12}$. It is very easy to see that the previous methods can be recovered by selecting $\sigma=0$ and $\sigma=\frac{1}{12}$, respectively. The resulting system is again a block nonlinear tridiagonal system which can be solved for the unknown vector $\boldsymbol{V}_{j, m}^{n+1}$ by any iterative solver, the fixed point method is adopted in this work.

\section{Fixed Point Method}

Since the generalized compact finite difference scheme (23) is nonlinear and implicit, an iterative method is needed to solve it. Two iterative algorithms are implemented to perform this job [11].

Algorithm 1

$$
\begin{aligned}
& \sigma \boldsymbol{V}_{j, m-1}^{n+1,(s+1)}+(1-2 \sigma) \boldsymbol{V}_{j, m}^{n+1,(s+1)}+\sigma \boldsymbol{V}_{j, m+1}^{n+1,(s+1)}+\frac{1}{2} r A\left[\boldsymbol{V}_{j, m-1}^{n+1,(s+1)}-2 \boldsymbol{V}_{j, m}^{n+1,(s+1)}+\boldsymbol{V}_{j, m+1}^{n+1,(s+1)}\right] \\
& =\sigma \boldsymbol{V}_{j, m-1}^{n}+(1-2 \sigma) \boldsymbol{V}_{j, m}^{n}+\sigma \boldsymbol{V}_{j, m+1}^{n}+\frac{1}{2} r A\left[\boldsymbol{V}_{j, m-1}^{n}-2 \boldsymbol{V}_{j, m}^{n}+\boldsymbol{V}_{j, m+1}^{n}\right] \\
& \quad+k\left[\sigma \boldsymbol{G}_{j, m-1}^{n,(s)}+(1-2 \sigma) \boldsymbol{G}_{j, m}^{n,(s)}+\sigma \boldsymbol{G}_{j, m+1}^{n,(s)}\right]
\end{aligned}
$$


where

$$
\boldsymbol{G}_{j, m}^{n,(s)}=\frac{1}{4}\left(\sum_{j=1}^{3}\left(\left|q_{j, m}^{n+1,(s)}\right|^{2}+\left|q_{j, m}^{n}\right|^{2}\right)\right) A\left[\boldsymbol{V}_{j, m}^{n+1,(s)}+\boldsymbol{V}_{j, m}^{n}\right], j=1,2,3
$$

where the superscript $s$ denotes the $s$ th iterate for solving the nonlinear system of equations for each iteration. The system in (24) can be solved by Crout's method, where we need only one LU factorization for the block tridiagonal matrix at the beginning, and the solutions of lower and upper bi-diagonal block systems at each iteration are required only.

\section{Algorithm 2}

$$
\begin{aligned}
& \sigma \boldsymbol{V}_{j, m-1}^{n+1,(s+1)}+(1-2 \sigma) \boldsymbol{V}_{j, m}^{n+1,(s+1)}+\sigma \boldsymbol{V}_{j, m+1}^{n+1,(s+1)}+\frac{1}{2} r A\left[\boldsymbol{V}_{j, m-1}^{n+1,(s+1)}-2 \boldsymbol{V}_{j, m}^{n+1,(s+1)}+\boldsymbol{V}_{j, m+1}^{n+1,(s+1)}\right] \\
& =\sigma \boldsymbol{V}_{j, m-1}^{n}+(1-2 \sigma) \boldsymbol{V}_{j, m}^{n}+\sigma \boldsymbol{V}_{j, m+1}^{n}+\frac{1}{2} r A\left[\boldsymbol{V}_{j, m-1}^{n}-2 \boldsymbol{V}_{j, m}^{n}+\boldsymbol{V}_{j, m+1}^{n}\right] \\
& +k\left[\sigma \boldsymbol{G}_{j, m-1}^{n,(s)}+(1-2 \sigma) \boldsymbol{G}_{j, m}^{n,(s)}+\sigma \boldsymbol{G}_{j, m+1}^{n,(s)}\right]
\end{aligned}
$$

where

$$
\boldsymbol{G}_{j, m}^{n,(s)}=\frac{1}{4} \sum_{j=1}^{3}\left(\left|q_{j, m}^{n+1,(s)}\right|^{2}+\left|q_{j, m}^{n}\right|^{2}\right) A\left[\boldsymbol{V}_{j, m}^{n+1,(s+1)}+\boldsymbol{V}_{j, m}^{n}\right], j=1,2,3,
$$

where the superscript $s$ denotes the $s$ th iterate for solving the nonlinear system of equations for each time. The block tridiagonal system (25) can be solved by Crout's method. Note that in this method we need to do factorization at each iteration. The initial iterate $\boldsymbol{U}_{m}^{n+1,(0)}$ can be chosen as $\boldsymbol{V}_{m}^{n+1,(0)}=\boldsymbol{V}_{m}^{n}$. We apply the iterative schemes till the following condition

$$
\left\|\boldsymbol{V}_{m}^{n,(s+1)}-\boldsymbol{V}_{m}^{n+1,(s)}\right\|_{\infty} \leq 10^{-6}
$$

is satisfied. The convergence of the iterative schemes, Algorithm 1 and Algorithm 2 is given in [11].

\section{Time Splitting Method}

In this work we are going to present the time splitting method for solving the 3-coupled nonlinear Schrödinger Equation (1). The basic idea in the time splitting method is to decompose the original problem into subproblems, which are simpler than the original problem and then to compose the approximate solution of the original problem by using the exact or approximate solutions of the subproblems in a given sequential order. To display this method for our system

$$
\begin{aligned}
& i q_{1 t}+q_{1 x x}+2\left(\left|q_{1}\right|^{2}+\left|q_{2}\right|^{2}+\left|q_{3}\right|^{2}\right) q_{1}=0, \\
& i q_{2 t}+q_{2 x x}+2\left(\left|q_{1}\right|^{2}+\left|q_{2}\right|^{2}+\left|q_{3}\right|^{2}\right) q_{2}=0, \\
& i q_{3 t}+q_{3 x x}+2\left(\left|q_{1}\right|^{2}+\left|q_{2}\right|^{2}+\left|q_{3}\right|^{2}\right) q_{3}=0,
\end{aligned}
$$

with initial conditions 


$$
q_{j}(x, 0)=g_{j}(x), x_{L} \leq x \leq x_{R}, j=1,2,3
$$

and the homogenous boundary conditions

$$
q_{1}(x, t)=q_{2}(x, t)=q_{3}(x, t)=0 \text { at } x=x_{L}, x_{R}
$$

The system in (26) can be written as

$$
\frac{\partial q_{1}}{\partial t}=(L+N) q_{1}, \frac{\partial q_{2}}{\partial t}=(L+N) q_{2}, \frac{\partial q_{3}}{\partial t}=(L+N) q_{3}
$$

where

$$
L=i \frac{\partial}{\partial x^{2}} \text { and } N=2 i\left(\left|q_{1}\right|^{2}+\left|q_{2}\right|^{2}+\left|q_{3}\right|^{2}\right) \text {. }
$$

We solve the system (27) from $t=t_{n}$ to $t=t_{n+1}$ in two splitting steps. We solve first the linear system equation

$$
\frac{\partial q_{j}}{\partial t}=i q_{j x x}, j=1,2,3
$$

with the homogenous Dirichlet boundary conditions

$$
q_{j}(x, t)=0 \text { at } x=x_{L}, x_{R},
$$

using the finite difference method for the time step $k$, followed by solving the nonlinear system

$$
\frac{\partial q_{j}}{\partial t}=i\left(\left|q_{1}\right|^{2}+\left|q_{2}\right|^{2}+\left|q_{3}\right|^{2}\right) q_{j}, j=1,2,3
$$

for the same time step. Equation (30) can be integrated exactly in time [15], the exact solution is

$$
\begin{gathered}
q_{j}(x, t)=\exp \left(i\left(\left|q_{1}\left(x, t_{n}\right)\right|^{2}+\left|q_{2}\left(x, t_{n}\right)\right|^{2}+\left|q_{3}\left(x, t_{n}\right)\right|^{2}\right)\left(t-t_{n}\right)\right) q_{j}, \\
t \geq t_{n}, x_{L} \leq x \leq x_{R}, j=1,2,3
\end{gathered}
$$

To apply this method in systematic way, we combine the splitting steps via the standard second order Strang splitting [21]. The flowchart of this method can be described by the following steps.

\section{Step 1: Solution of the nonlinear problem}

$$
\begin{aligned}
q_{j, m}^{(1)}(x, t) & =\exp \left(i\left(\left|q_{1, m}^{n}\right|^{2}+\left|q_{2, m}^{n}\right|^{2}+\left|q_{3, m}^{n}\right|^{2}\right)\left(\frac{k}{2}\right)\right) q_{j, m}^{n}, \\
q_{j, 0}^{(1)} & =q_{j, M}^{(1)}=0, m=1,2, \cdots, M-1, j=1,2,3 .
\end{aligned}
$$

\section{Step 2: Solution of the linear problem}

The solution of the linear can be obtained using the generalized difference scheme

$$
\begin{aligned}
& \left(\sigma I+\frac{1}{2} r A\right) q_{j, m-1}^{(2)}+[(1-2 \sigma) I-r A] q_{j, m}^{(2)}+\left(\sigma I+\frac{1}{2} r A\right) q_{j, m+1}^{(2)} \\
& =\left(\sigma I-\frac{1}{2} r A\right) q_{j, m-1}^{(1)}+[(1-2 \sigma) I+r A] q_{j, m}^{(1)}+\left(\sigma I-\frac{1}{2} r A\right) q_{j, m+1}^{(1)},
\end{aligned}
$$




$$
q_{j, 0}^{(2)}=q_{j, M}^{(2)}=0, m=1,2, \cdots, M-1, j=1,2,3 .
$$

The solution of this system can be obtained by solving linear block tridiagonal system with constant coefficients using Crouts method, and this can be executed in very efficient way.

Step 3: Solution of the nonlinear problem

$$
\begin{gathered}
q_{j, m}^{n+1}(x, t)=\exp \left(i\left(\left|q_{1, m}^{(2)}\right|^{2}+\left|q_{2, m}^{(2)}\right|^{2}+\left|q_{3, m}^{(2)}\right|^{2}\right)\left(\frac{k}{2}\right)\right) q_{j, m}^{(2)}, \\
q_{j, 0}^{n+1}=q_{j, M}^{n+1}=0, m=1,2, \cdots, M-1, j=1,2,3 .
\end{gathered}
$$

The numerical scheme is of second order accuracy in time, second and fourth order in space for $\sigma=0$ and $\sigma=\frac{1}{12}$, respectively. It is unconditionally stable and con-

served the conserved quantities exactly [15] [20] [23]. We denote this method by the time splitting finite difference method by (TSFDM).

\section{Numerical Results}

In this section, we conduct some typical numerical examples to verify the accuracy, conservation laws, computational efficiency and some physical interaction phenomena described by 3-coupled nonlinear Schrödinger equations.

\subsection{Single Soliton}

In this test, we choose the initial condition as

$$
\begin{aligned}
& q_{j}(x, 0)=A_{j} k_{1 R} \exp \left(i k_{1 I} x\right) \operatorname{sech}\left(k_{1 R} x+\frac{R}{2}\right), \\
& A_{j}=\frac{\alpha_{j}}{\Delta}, \mathrm{e}^{R}=\frac{\Delta^{2}}{\left(k_{1}+k_{1}^{*}\right)^{2}}, \Delta^{2}=\sum_{j=1}^{3}\left|\alpha_{j}\right|^{2}, j=1,2,3 .
\end{aligned}
$$

The following set of parameters are used

$$
\begin{gathered}
h=0.1, k=0.01, x_{l}=-30, t=0,0.2, \cdots, 10 \\
\alpha_{1}=1+i, \alpha_{2}=0.8+0.8 i, \alpha_{3}=0.5+0.5 i, k_{1}=1+0.5 i
\end{gathered}
$$

The error and the conserved quantities as well as the execution time for all methods are given in Tables 1-6, we have noticed that all method are conserved the conserved quantities exactly and for accuracy the credit goes to the fourth order scheme $\left(\sigma=\frac{1}{12}\right)$. The profile of $\left|q_{1}\right|,\left|q_{2}\right|$ and $\left|q_{3}\right|$ at different times are displayed in Figures 1-3. respectively.

To test the convergent rate in space and time of the proposed schemes. We define the $L_{\infty}$ error norm by

$$
L_{\infty}(h)=\left\|\boldsymbol{E}_{m}^{n}\right\|=\max _{1 \leq m \leq M}\left|u_{1, m}^{n}-U_{1, m}^{n}\right|,
$$

where $u_{1, m}^{n}$ and $U_{1, m}^{n}$ are respectively the exact and the numerical solution at the grid 
Table 1. Second order scheme $(\sigma=0)$ (Algorithm 1$), \mathrm{cpu}=2.28 \mathrm{sec}$.

\begin{tabular}{cccccc}
\hline $\mathrm{T}$ & $L_{\infty}$ & $L_{2}$ & $l_{1}\left(q_{1}\right)$ & $l_{2}\left(q_{2}\right)$ & 0.677249 \\
\hline 0.0 & 0.000000 & 0.000000 & 1.058201 & 0.677249 & 0.264550 \\
2.0 & 0.004791 & 0.007296 & 1.058201 & 0.677249 & 0.264550 \\
4.0 & 0.009952 & 0.015365 & 1.058201 & 0.677249 & 0.264550 \\
6.0 & 0.014046 & 0.021150 & 1.058201 & 0.677249 & 0.264550 \\
8.0 & 0.018731 & 0.028129 & 1.058201 & 0.677249 & 0.264550 \\
\hline
\end{tabular}

Table 2. Fourth order scheme $(\sigma=1 / 12)$ (Algorithm 1$), \mathrm{cpu}=2.27 \mathrm{sec}$.

\begin{tabular}{cccccc}
\hline $\mathrm{T}$ & $L_{\infty}$ & $L_{2}$ & $l_{1}\left(q_{1}\right)$ & $l_{2}\left(q_{2}\right)$ & 0.677249 \\
\hline 0 & 0.000000 & 0.000000 & 1.058201 & 0.677249 & 0.264550 \\
2.0 & 0.000035 & 0.000049 & 1.058201 & 0.677249 & 0.264550 \\
4.0 & 0.000074 & 0.000107 & 1.058201 & 0.677249 & 0.264550 \\
6.0 & 0.000109 & 0.000154 & 1.058201 & 0.677249 & 0.264550 \\
8.0 & 0.000145 & 0.000205 & 1.058201 & 0.677249 & 0.264550 \\
10.0 & 0.000183 & 0.000260 & 1.058201 & 0 \\
\hline
\end{tabular}

Table 3. Second order scheme $(\sigma=0)$ (Algorithm 2$), \mathrm{cpu}=3.13 \mathrm{sec}$.

\begin{tabular}{cccccc}
\hline $\mathrm{T}$ & $L_{\infty}$ & $L_{2}$ & $l_{1}\left(q_{1}\right)$ & $l_{2}\left(q_{2}\right)$ & 0.677249 \\
0.0 & 0.000000 & 0.000000 & 1.058201 & 0.677249 & 0.264550 \\
2.0 & 0.004791 & 0.007296 & 1.058201 & 0.677249 & 0.264550 \\
4.0 & 0.009951 & 0.015365 & 1.058201 & 0.677249 & 0.264550 \\
6.0 & 0.014046 & 0.021149 & 1.058201 & 0.677249 & 0.264550 \\
8.0 & 0.018730 & 0.028128 & 1.058201 & 0.677249 & 0.264550 \\
\hline
\end{tabular}

Table 4. Fourth order scheme $(\sigma=1 / 12)$ (Algorithm 2), cpu $=3.14 \mathrm{sec}$.

\begin{tabular}{cccccc}
\hline $\mathrm{T}$ & $L_{\infty}$ & $L_{2}$ & $l_{1}\left(q_{1}\right)$ & $l_{2}\left(q_{2}\right)$ & 0.677249 \\
\hline 0 & 0.000000 & 0.000000 & 1.058201 & 0.677249 & 0.264550 \\
2.0 & 0.000035 & 0.000049 & 1.058201 & 0.677249 & 0.264550 \\
4.0 & 0.000074 & 0.000107 & 1.058201 & 0.677249 & 0.264550 \\
6.0 & 0.000109 & 0.000153 & 1.058201 & 0.677249 & 0.264550 \\
8.0 & 0.000144 & 0.000203 & 1.058201 & 0.677249 & 0.264550
\end{tabular}


Table 5. TSFDM $(\sigma=0), \mathrm{cpu}=1.15 \mathrm{sec}$.

\begin{tabular}{cccccc}
\hline $\mathrm{T}$ & $L_{\infty}$ & $L_{2}$ & $l_{1}\left(q_{1}\right)$ & $l_{2}\left(q_{2}\right)$ & 0.677249 \\
\hline 0.0 & 0.000000 & 0.000000 & 1.058201 & 0.677249 & 0.264550 \\
2.0 & 0.004977 & 0.007588 & 1.058201 & 0.677249 & 0.264550 \\
4.0 & 0.010348 & 0.015978 & 1.058201 & 0.677249 & 0.264550 \\
6.0 & 0.014609 & 0.022000 & 1.058201 & 0.677249 & 0.264550 \\
8.0 & 0.019486 & 0.029261 & 1.058201 & 0.677249 & 0.264550 \\
10.0 & 0.024908 & 0.037654 & 1.058201 & 0.264550 & \\
\hline
\end{tabular}

Table 6. TSFDM $(\sigma=1 / 12), \mathrm{cpu}=1.41 \mathrm{sec}$.

\begin{tabular}{|c|c|c|c|c|c|}
\hline $\mathrm{T}$ & $L_{\infty}$ & $L_{2}$ & $l_{1}\left(q_{1}\right)$ & $l_{2}\left(q_{2}\right)$ & $l_{3}\left(q_{3}\right)$ \\
\hline 0.0 & 0.000000 & 0.000000 & 1.058201 & 0.677249 & 0.264550 \\
\hline 2.0 & 0.000160 & 0.000251 & 1.058201 & 0.677249 & 0.264550 \\
\hline 4.0 & 0.000326 & 0.000512 & 1.058201 & 0.677249 & 0.264550 \\
\hline 6.0 & 0.000461 & 0.000708 & 1.058201 & 0.677249 & 0.264550 \\
\hline 8.0 & 0.000603 & 0.000936 & 1.058201 & 0.677249 & 0.264550 \\
\hline 10.0 & 0.000773 & 0.001203 & 1.058201 & 0.677249 & 0.264550 \\
\hline
\end{tabular}

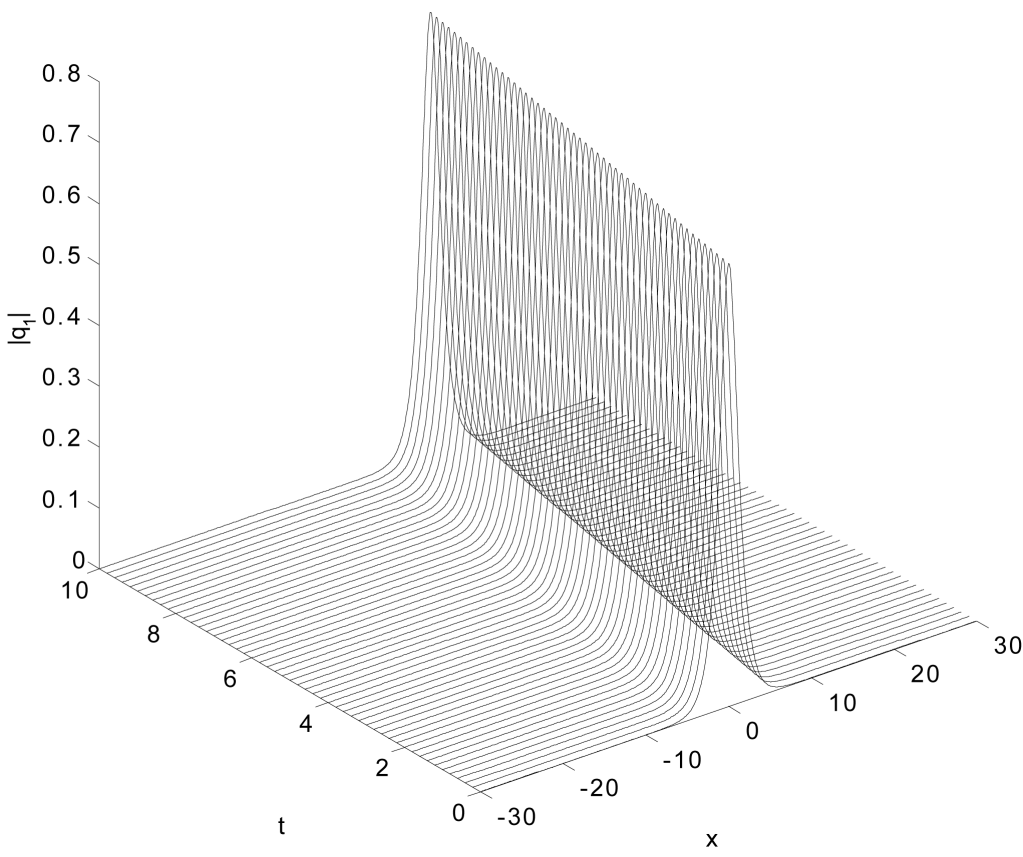

Figure 1. Single soliton: $\left|q_{1}\right|$.

point $\left(x_{m}, t_{n}\right)$. In this experiment, we take $T=1$. The convergent rate "order" is calculated by the formula

$$
\operatorname{order}(\text { rate of convergent in space })=\frac{\ln \left(\frac{L_{\infty}\left(h_{1}\right)}{L_{\infty}\left(h_{2}\right)}\right)}{\ln \left(\frac{h_{1}}{h_{2}}\right)}
$$




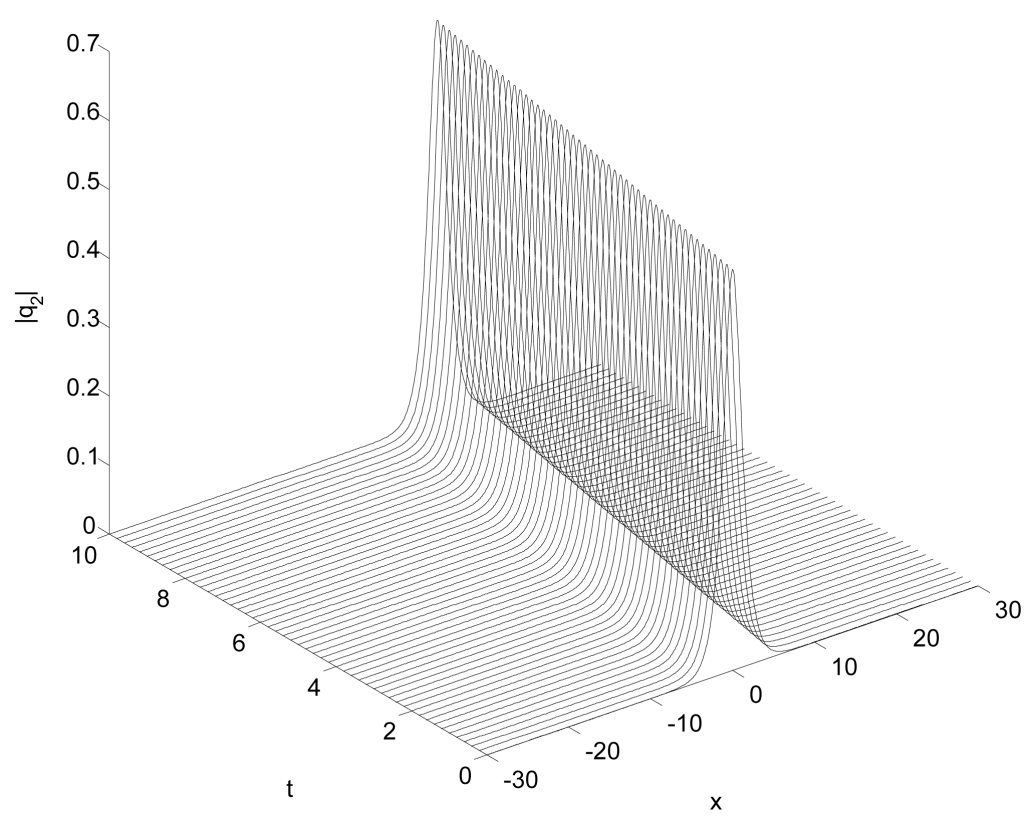

Figure 2. Single soliton: $\left|q_{2}\right|$.

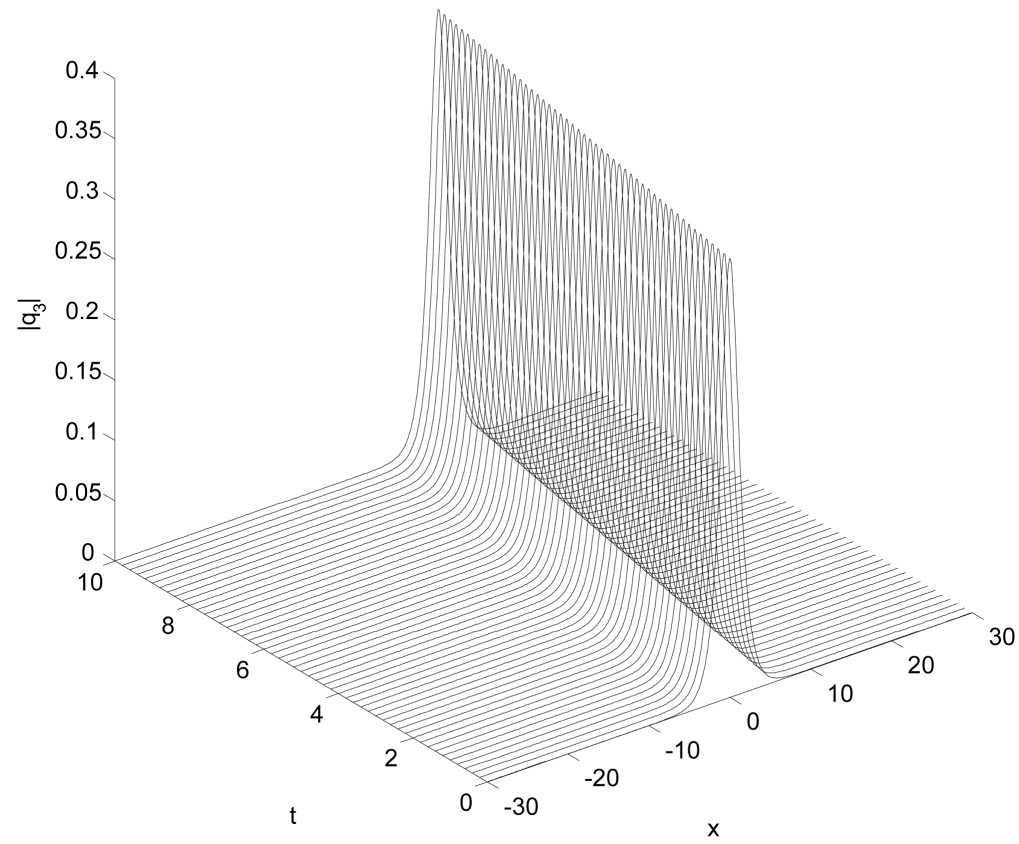

Figure 3. Single soliton: $\left|q_{3}\right|$.

$$
\operatorname{order}(\text { rate of convergent in time })=\frac{\ln \left(\frac{L_{\infty}\left(k_{1}\right)}{L_{\infty}\left(k_{2}\right)}\right)}{\ln \left(\frac{k_{1}}{k_{2}}\right)}
$$

To calculate the convergent rate in space, we take the time step $k$ sufficiently small and the error from temporal truncation is relatively small $k=5 \times 10^{-4}$. From Table 7 , 
Table 7. Spatial order of convergent with $k=0.0005$ at $T=1$.

\begin{tabular}{ccccc}
\hline $\mathrm{h}$ & $L_{\infty}(\mathrm{Re})$ & Rate & $L_{\infty}(\operatorname{Im})$ & Rate \\
\hline 0.4 & 0.002548 & - & 0.002205 & - \\
0.2 & 0.000166 & 3.936 & 0.000192 & 4.095 \\
0.1 & 0.000010 & 4.095 & 0.000008 & 4.011 \\
0.05 & 0.000001 & 3.333 & 0.000001 & 3.000 \\
\hline
\end{tabular}

we can easily that the rate of convergent is 4 as we claim in this work.

To check the temporal convergent rate, we fix the spatial step $h$ small enough so that the truncation from space is negligible such as $h=0.01$. The results are given in Table 8 which indicate that the order is 2 as we claim in the text.

\subsection{Interaction of Two Solitons}

To study the interaction of two solitons with different parameters, we choose the initial condition as a sum of two single solitons of the form

$$
q_{j}(x, t=0)=q_{j}^{(1)}(x, 0)+q_{j}^{(2)}(x, 0), j=1,2,3,
$$

where

$$
\begin{aligned}
& q_{j}^{(1)}(x, 0)=A_{j}^{(1)} k_{1 R} \exp \left(i k_{1 I}\left(x+x_{0}\right)\right) \operatorname{sech}\left(k_{1 R}\left(x+x_{0}\right)+\frac{R}{2}\right), \\
& q_{j}^{(2)}(x, 0)=A_{j}^{(2)} k_{1 R} \exp \left(i k_{1 I}\left(x-x_{0}\right)\right) \operatorname{sech}\left(k_{1 R}\left(x-x_{0}\right)+\frac{R}{2}\right) .
\end{aligned}
$$

For all examples in the case of interaction, we choose the set of parameters

$$
\begin{gathered}
h=0.1, k=0.01, x_{L}=-50, x_{R}=50, x_{0}=25, \\
k_{1}=1+0.8 i, k_{2}=1-0.4 i,
\end{gathered}
$$

together with different values of $\left\{\alpha_{1}^{(j)}, \alpha_{2}^{(j)}, j=1,2,3\right\}$ for each test. We will study the dynamics of the following cases

Test 1: Two Solitons (with Pure Imaginary Parameters)

In this test we will consider the two set of parameters (equal and different )

$$
\alpha_{1}^{(1)}=\alpha_{2}^{(1)}=5 i, \alpha_{1}^{(2)}=\alpha_{2}^{(2)}=3.5 i, \alpha_{1}^{(3)}=\alpha_{2}^{(3)}=i
$$

and

$$
\alpha_{1}^{(1)}=5 i, \alpha_{1}^{(2)}=3.5 i, \alpha_{1}^{(3)}=i, \alpha_{2}^{(1)}=2 i, \alpha_{2}^{(2)}=2.5 i, \alpha_{2}^{(3)}=i
$$

For the first set of parameters (36), we have noticed that the amplitudes of soliton 1 and soliton 2 before the interaction are $S_{1}=[0.8081,0.5657,0.166]$ and $S_{2}=[0.8081,0.5657,0.166]$ remain unchanged after the interaction which means the interaction is elastic and this scenario is displayed in Figures 4-6.

For the second set of parameters (37), we have noticed that the amplitudes of soliton 1 and soliton 2 before the interaction are $S_{1}=[0.8081,0.5657,0.166]$ and 
Table 8. Temporal order of convergent with $h=0.01$ at $T=1$.

\begin{tabular}{ccccc}
\hline$h$ & $L_{\infty}(\mathrm{Re})$ & Rate & $L_{\infty}(\operatorname{Im})$ & Rate \\
\hline 0.2 & 0.009658 & - & 0.006826 & - \\
0.1 & 0.002426 & 1.993 & 0.001653 & 2.046 \\
0.05 & 0.000610 & 1.992 & 0.000415 & 1.993 \\
0.025 & 0.000150 & 1.986 & 0.000104 & 1.997 \\
\hline
\end{tabular}

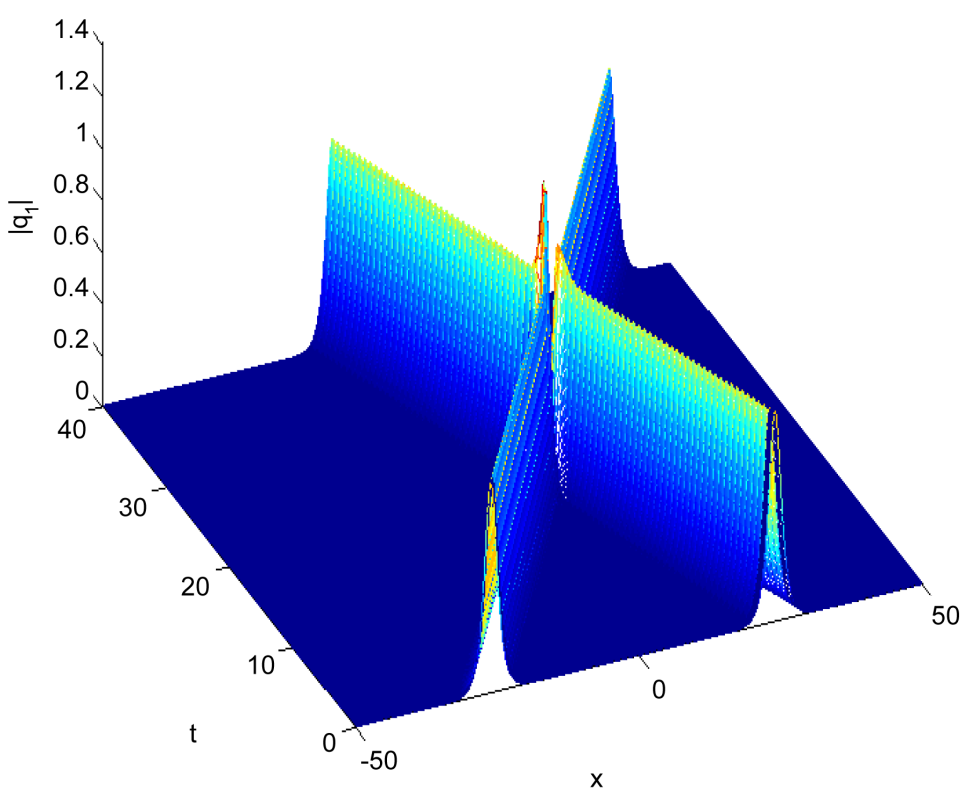

Figure 4. Elastic interaction with equal pure imaginary parameters $\left|q_{1}\right|$.

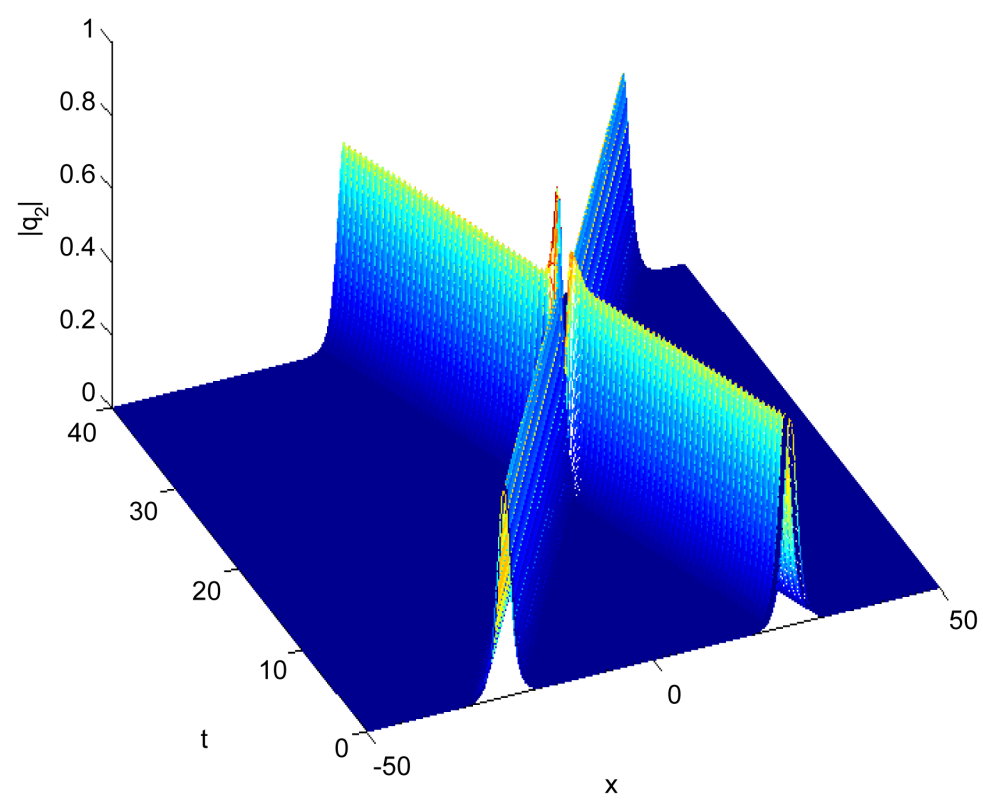

Figure 5. Elastic interaction with equal pure imaginary parameters $\left|q_{2}\right|$. 


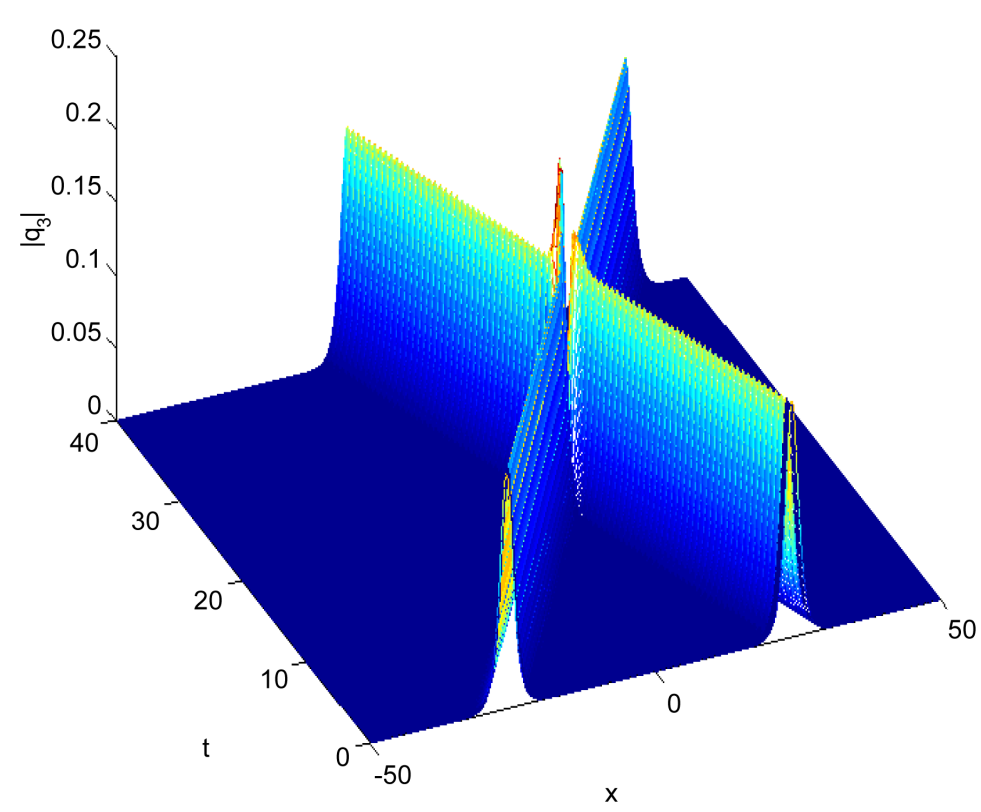

Figure 6. Elastic interaction with equal pure imaginary parameters $\left|q_{3}\right|$.

$S_{2}=[0.5962,0.7452,0.2981]$ change to $S_{1}=[0.6631,0.6986,0.2664]$ and $S_{2}=[0.7542,0.6222,0.2097]$ after the interaction, the collision mechanism can be described as follows

$$
\begin{aligned}
& S_{1} \Rightarrow\left[q_{1}(\text { Suppressed }), q_{2}(\text { Enhanced }), q_{3}(\text { Enhanced })\right], \\
& S_{2} \Rightarrow\left[q_{1}(\text { Enhanced }), q_{2}(\text { Suppressed }), q_{3}(\text { Suppressed })\right],
\end{aligned}
$$

and in this case we have inelastic collision and we display this scenario in Figures 7-9.

\subsection{Test 2: (Pure Real Parameters)}

In this test, we choose two different test of parameters

$$
\alpha_{1}^{(1)}=5, \alpha_{1}^{(2)}=3.5, \alpha_{1}^{(3)}=1, \alpha_{2}^{(1)}=10, \alpha_{2}^{(2)}=7, \alpha_{2}^{(3)}=2
$$

and

$$
\alpha_{1}^{(1)}=5, \alpha_{1}^{(2)}=3.5, \alpha_{1}^{(3)}=1, \alpha_{2}^{(1)}=2, \alpha_{2}^{(2)}=2.5, \alpha_{2}^{(3)}=0.4
$$

For the first set of parameters (38), we have noticed that the amplitudes of soliton 1 and soliton 2 before the interaction are $S_{1}=[0.8081,0.5657,0.166]$ and $S_{2}=[0.8081,0.5657,0.166]$ remain unchanged after the interaction which means the interaction is elastic and this scenario is displayed in Figures 10-12.

For the second set of parameters (39), we have noticed that the amplitudes of soliton 1 and soliton 2 before the interaction are $S_{1}=[0.8081,0.5657,0.166]$ and $S_{2}=[0.6197,0.77747,0.1239]$ change to $S_{1}=[0.6779,0.7219,0.1356]$ and $S_{2}=[0.7600,0.6315,0.1520]$ after the interaction, this means the collision mechanism is inelastic and can be given as

$$
\left.\left.S_{1} \Rightarrow\left[q_{1} \text { (Suppressed }\right), q_{2}(\text { Enhanced }), q_{3} \text { (Suppressed }\right)\right],
$$




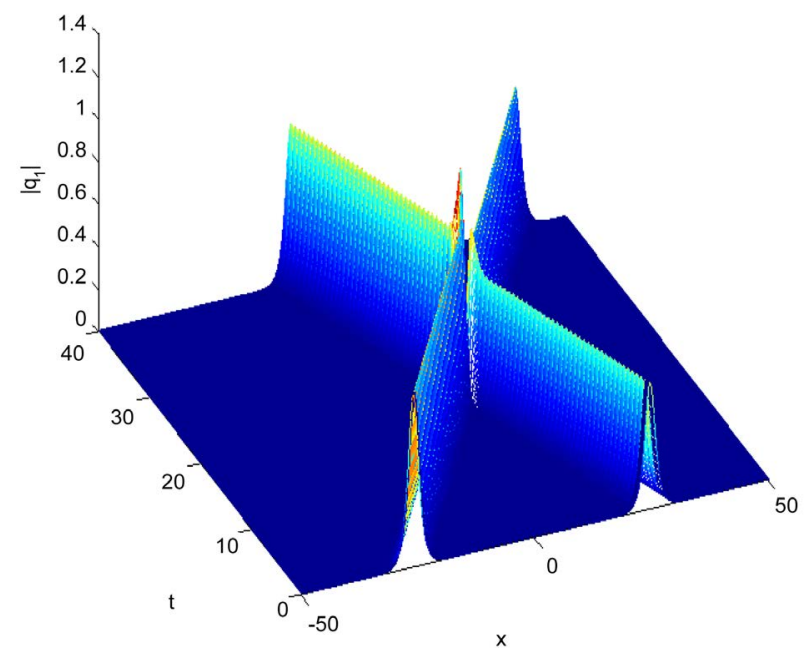

Figure 7. Inelastic interaction with different pure imaginary parameters $\left|q_{1}\right|$.

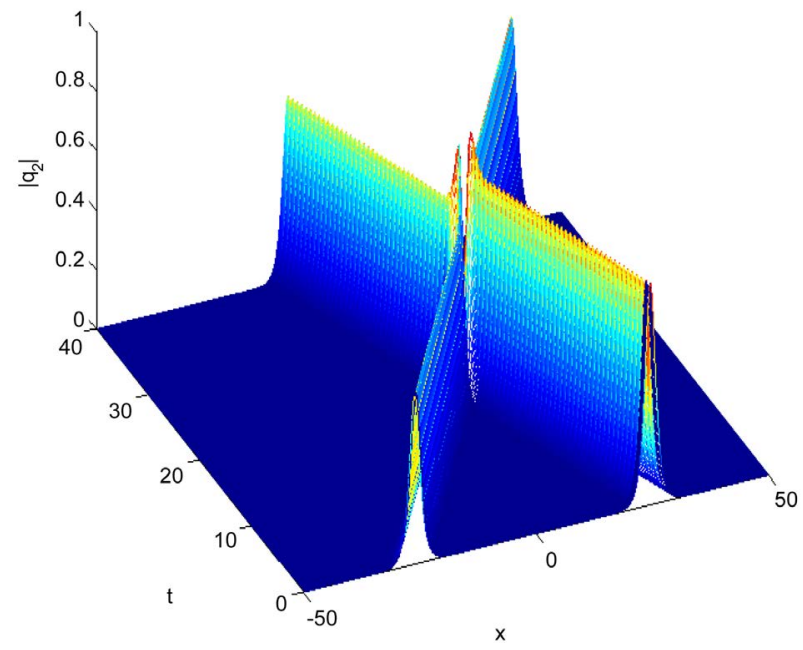

Figure 8. Inelastic interaction with different pure imaginary parameters $\left|q_{2}\right|$.

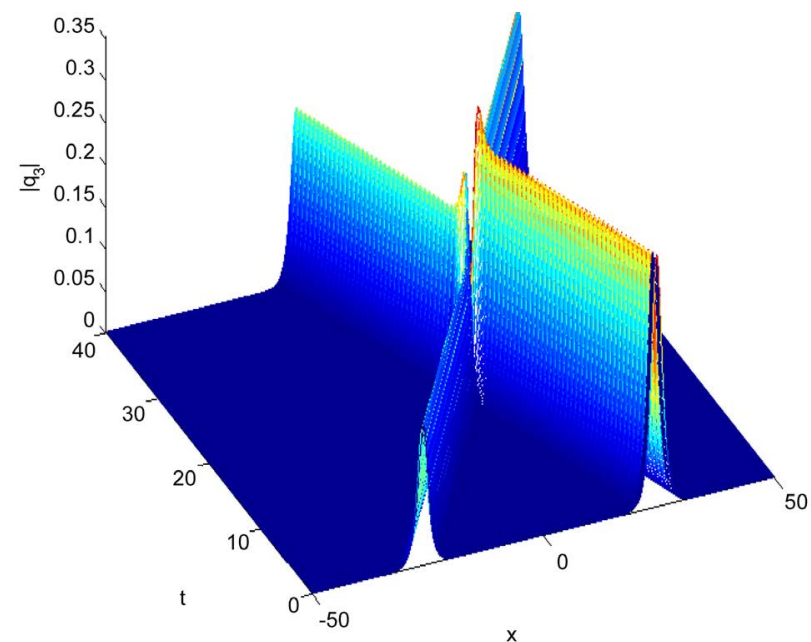

Figure 9. Inelastic interaction with different pure imaginary parameters $\left|q_{3}\right|$. 


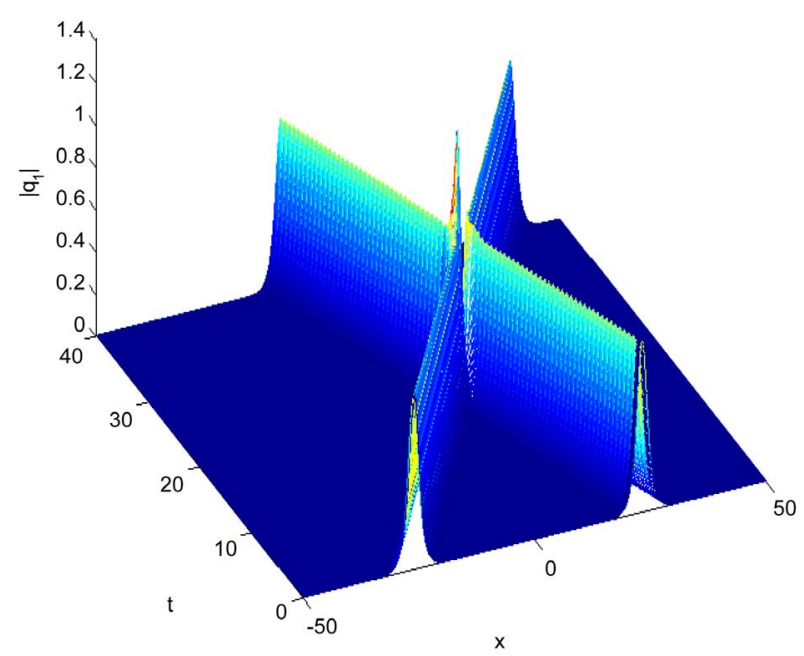

Figure 10. Elastic interaction with pure real parameters $q_{1}$.

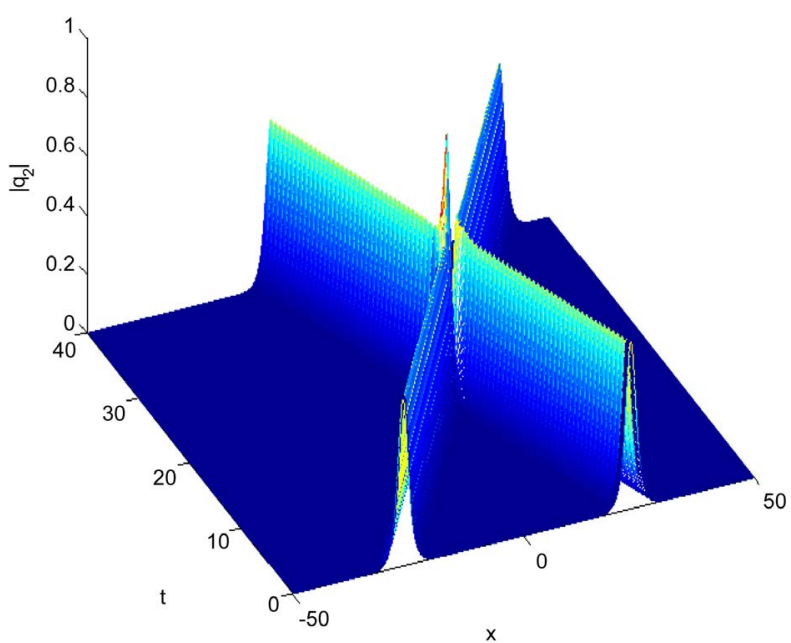

Figure 11. Elastic interaction with pure real parameters $q_{2}$.

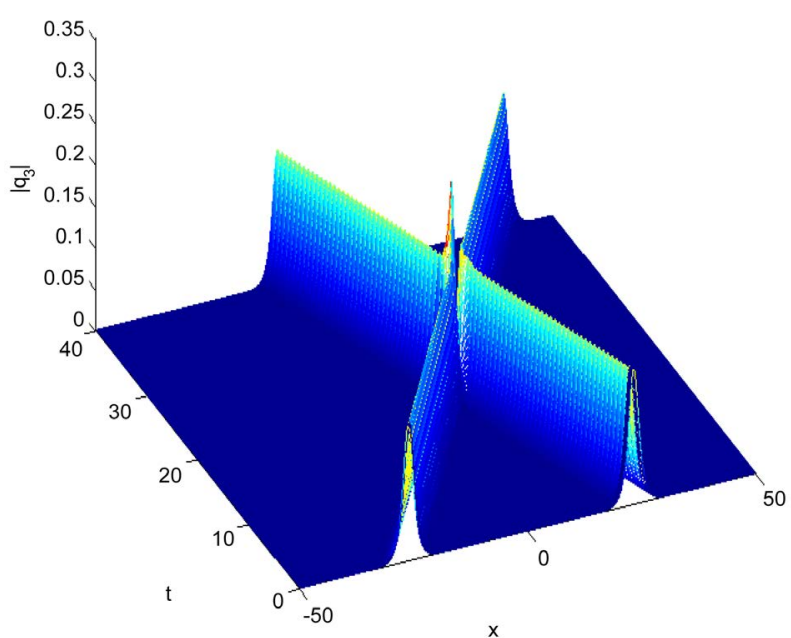

Figure 12. Elastic interaction with pure real parameters $q_{3}$. 


$$
S_{2} \Rightarrow\left[q_{1}(\text { Enhanced }), q_{2}(\text { Suppressed }), q_{3}(\text { Enhanced })\right],
$$

we display the interaction scenario in Figures 13-15.

\subsection{Test 3: Soliton Interaction (Nonzero Real and Imaginary Parts)}

In this test, we choose the two sets of parameters

$$
\begin{aligned}
& \alpha_{1}^{(1)}=1+i, \alpha_{1}^{(2)}=1+i, \alpha_{1}^{(3)}=1+i, \\
& \alpha_{2}^{(1)}=0.5+0.5 i, \alpha_{2}^{(2)}=0.5+0.5 i, \alpha_{2}^{(3)}=0.5+0.5 i,
\end{aligned}
$$

and

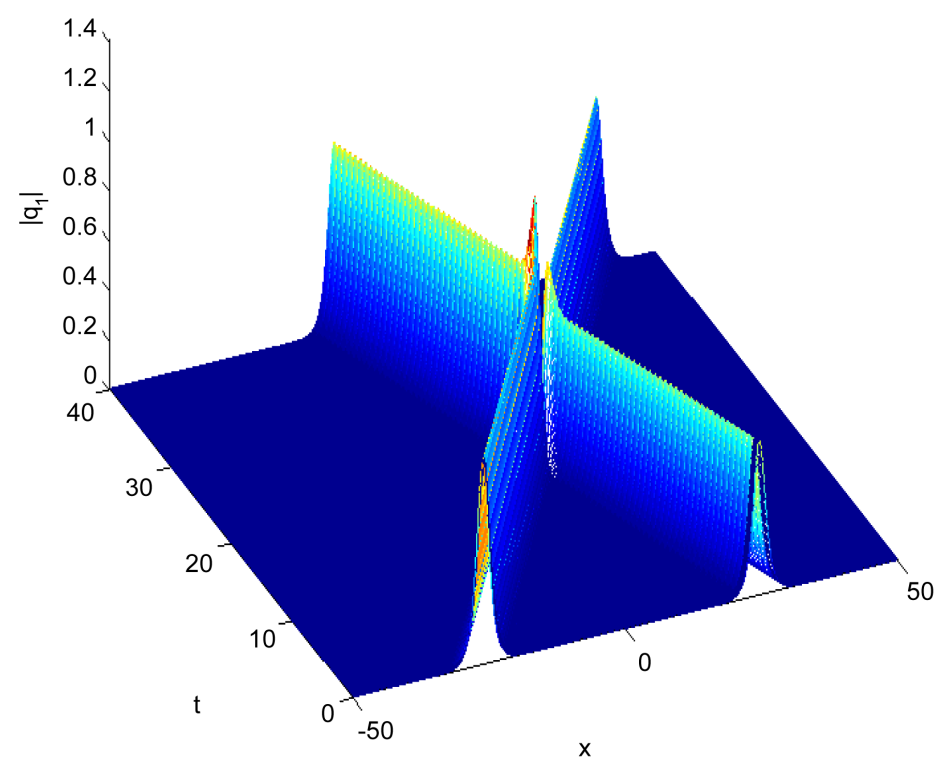

Figure 13. Inelastic interaction with pure real diøerent parameters $q_{1}$.

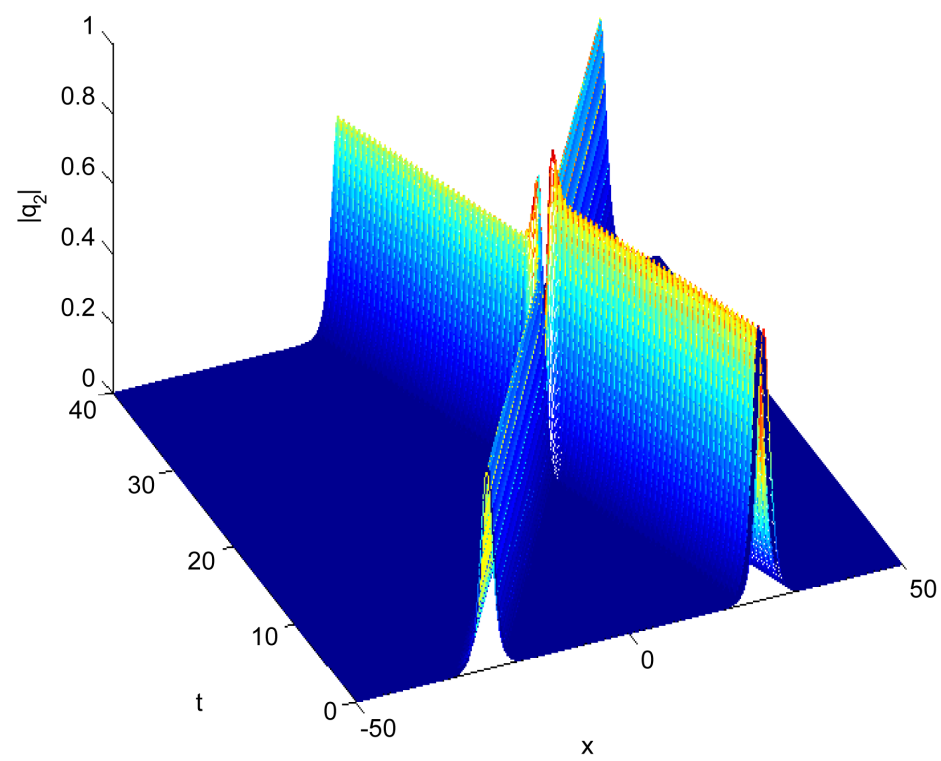

Figure 14. Inelastic interaction with pure real different parameters $q_{2}$. 


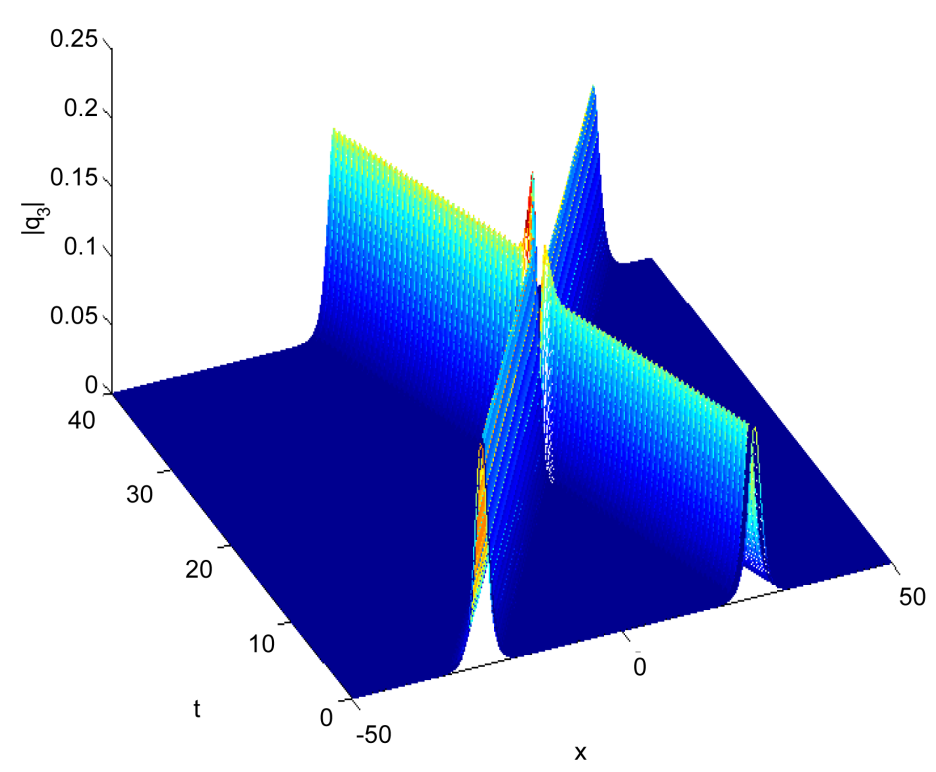

Figure 15. Inelastic interaction with pure real different parameters $q_{3}$.

$$
\begin{aligned}
& \alpha_{1}^{(1)}=1+i, \alpha_{1}^{(2)}=1+i, \alpha_{1}^{(3)}=1+i, \\
& \alpha_{2}^{(1)}=3+i, \alpha_{2}^{(2)}=1+2 i, \alpha_{2}^{(3)}=4+5 i .
\end{aligned}
$$

For the first set of parameters (40), we have noticed that the amplitudes of soliton 1 and soliton 2 before the interaction are $S_{1}=[0.5773,0.5773,0.5773]$ and $S_{2}=[0.5772,0.5772,0.5772]$ remain unchanged after the interaction which means the interaction is elastic and this scenario is displayed in Figures 16-18.

For the second set of parameters, we have noticed that the amplitudes of soliton 1 and soliton 2 before the interaction are $S_{1}=[0.5773,0.5773,0.5773]$ and $S_{2}=[0.4225,0.2988,0.8555]$ change to $S_{1}=[0.5743,0.3414,0.7435]$ and $S_{2}=[0.4259,0.5525,0.7149]$ after the interaction, the collision mechanism can be given as follows

$$
\begin{aligned}
& S_{1} \Rightarrow\left[q_{1}, q_{2}(\text { Suppressed }), q_{3}(\text { Enhanced })\right], \\
& S_{2} \Rightarrow\left[q_{1}, q_{2}(\text { Enhanced }), q_{3}(\text { Suppressed })\right],
\end{aligned}
$$

and in this case we have inelastic collision and we display this scenario in Figures 19-21.

\section{Conclusion}

In this work, we have derived different methods for solving the 3-coupled nonlinear Schrödinger equation using finite difference method and time splitting method with finite difference methods. All schemes are unconditionally stable and highly accurate and conserve the conserved quantities exactly. The interaction of two solitons is discussed in details for different parameters. We have noticed that to have elastic interaction the following constraint 


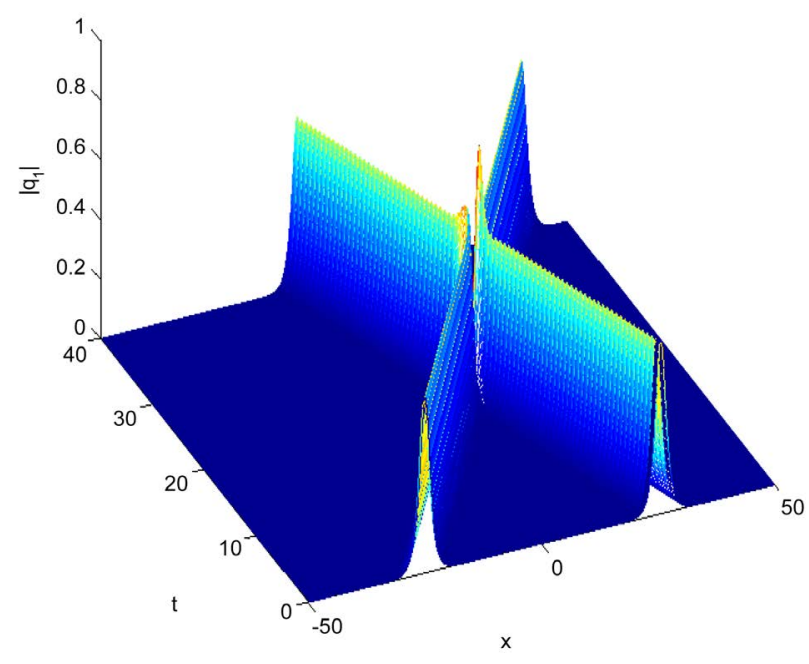

Figure 16. Elastic interaction with non zero real and imaginary parameters for $q_{1}$.

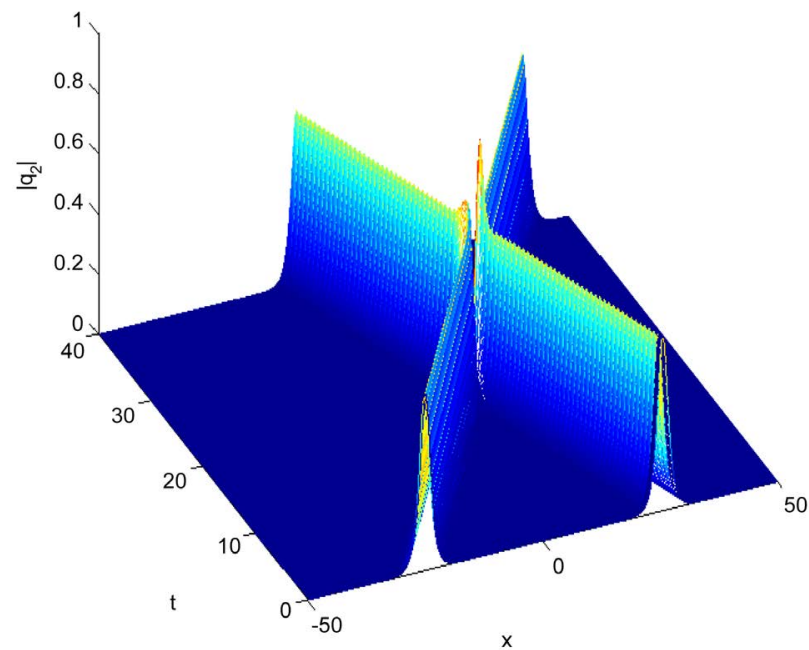

Figure 17. Elastic interaction with non zero real and imaginary parameters for $q_{2}$.

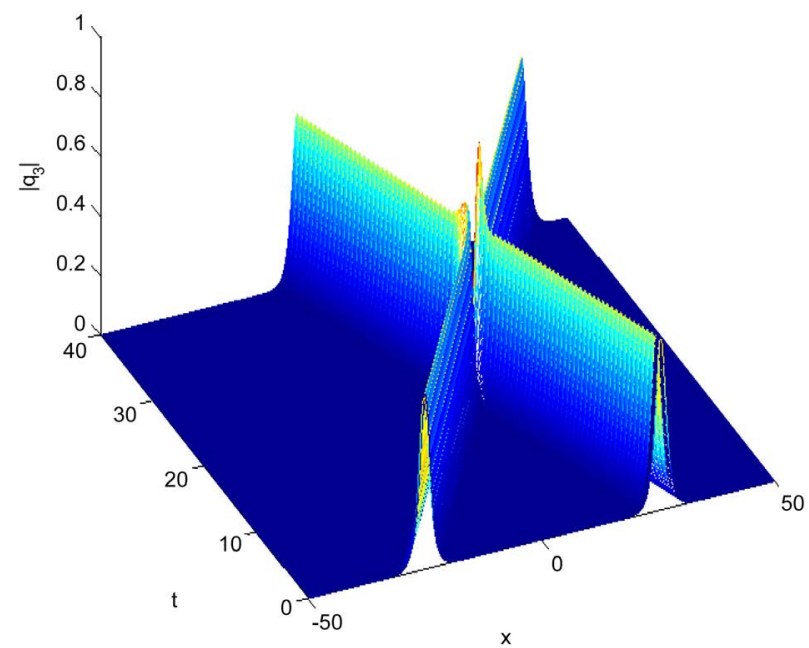

Figure 18. Elastic interaction with non zero real and imaginary parameters for $q_{3}$. 


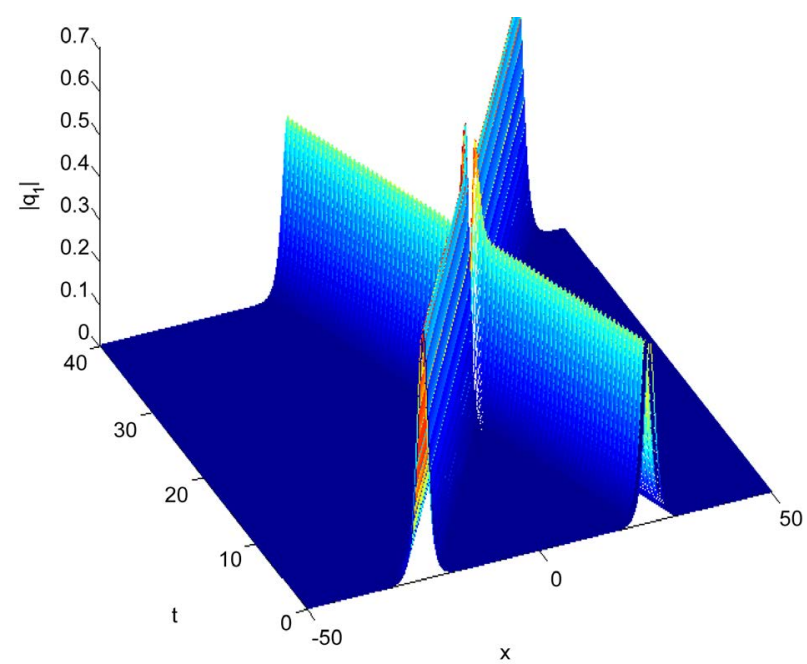

Figure 19. Inelastic interaction with non zero real and imaginary different parameters for $q_{1}$.

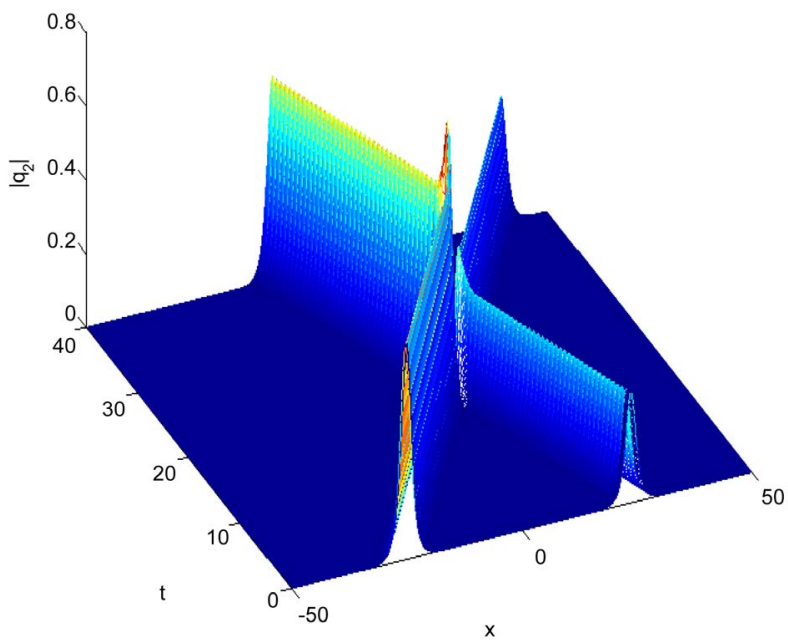

Figure 20. Inelastic interaction with non zero real and imaginary different parameters for $q_{2}$.

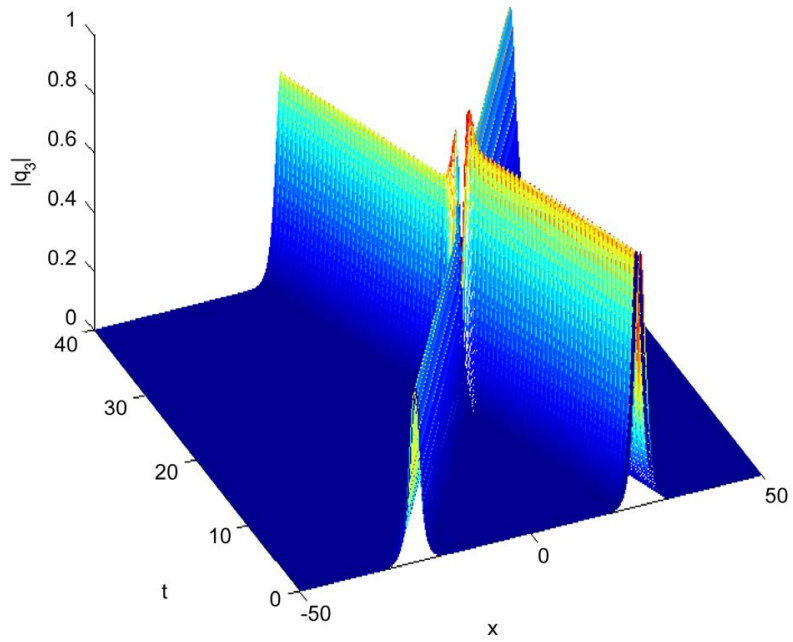

Figure 21. Inelastic interaction with non zero real and imaginary different parameters for $q_{3}$. 


$$
\frac{\alpha_{1}^{(1)}}{\alpha_{2}^{(1)}}=\frac{\alpha_{1}^{(2)}}{\alpha_{2}^{(2)}}=\frac{\alpha_{1}^{(3)}}{\alpha_{2}^{(3)}},
$$

must be satisfied, and for other values the interaction is inelastic, and different behaviors occur (enhancement,suppression) in the amplitude of each soliton. This behavior is in agreement with [1] [2] [3] [4]. The derived methods can be used to solve similar nonlinear problems.

\section{References}

[1] Kanna, T. and Lakshmanan, M. (2003) Exact Soliton Solutions of Coupled Nonlinear Schrödinger Equation: Shape-Changing Collisions, Logic Gates and Partially Coherent Soliton. Physical Review E, 67, Article ID: 046617. http://dx.doi.org/10.1103/physreve.67.046617

[2] Kanna, T. and Lakshmanan, M. (2001) Exact Soliton Solutions, Shape Changing Collisions and Partially Coherent Solitons in Coupled Nonlinear Schrödinger Equations. Physical Review Letter, 86, 5043-5046. http://dx.doi.org/10.1103/PhysRevLett.86.5043

[3] Lü, X. and Lin, F. (2016) Soliton Excitations and Shape-Changing Collisions in Alpha Helical Protines with Interspine Coupling at Higher Order. Communications in Nonlinear Science and Numerical Simulation, 32, 241-261. http://dx.doi.org/10.1016/j.cnsns.2015.08.008

[4] Lü, X. (2014) Bright-Soliton Collisions with Shape Change by Intensity Redistribution for the Coupled Sasa-Satsuma System in the Optical Fiber Communications. Communications in Nonlinear Science and Numerical Simulation, 19, 3969-3987.

http://dx.doi.org/10.1016/j.cnsns.2014.03.013

[5] Ismail, M.S. (2008) Numerical Solution of Coupled Nonlinear Schrödinger Equation by Galerkin Method. Mathematics and Computers in Simulation, 78, 532-547.

http://dx.doi.org/10.1016/j.matcom.2007.07.003

[6] Ismail, M.S., Al-Basyouni, K.S. and Aydin, A. (2015) Conservative Finite Difference Schemes for the Chiral Nonlinear Schrödinger Equation. Boundary Value Problem, 2015, 89. http://dx.doi.org/10.1186/s13661-015-0350-4

[7] Ismail, M.S. (2008) A Fourth Order Explicit Schemes for the Coupled Nonlinear Schrödinger Equation. Applied Mathematics and Computation, 196, 273-284. http://dx.doi.org/10.1016/j.amc.2007.05.059

[8] Ismail, M.S. and Taha, T.R. (2001) Numerical Simulation of Coupled Nonlinear Schrödinger Equation. Mathematics and Computers in Simulation, 56, 547-562.

http://dx.doi.org/10.1016/S0378-4754(01)00324-X

[9] Ismail, M.S. and Alamri, S.Z. (2004) Highly Accurate Finite Difference Method for Coupled Nonlinear Schrödinger Equation. International Journal of Computer Mathematics, 81, 333351. http://dx.doi.org/10.1080/00207160410001661339

[10] Ismail, M.S. and Taha, T.R. (2007) A Linearly Implicit Conservative Scheme for the Coupled Nonlinear Schrödinger Equation. Mathematics and Computers in Simulation, 74, 302-311. http://dx.doi.org/10.1016/j.matcom.2006.10.020

[11] Hu, X. and Zhang, L. (2014) Conservative Compact Difference Schemes for the Coupled Nonlinear Schrödinger System. Numerical Methods for Partial Differential Equations, 30, 749-772. http://dx.doi.org/10.1002/num.21826

[12] Kong, L., Ji, L. and Zhu, P. (2015) Compact and Efficient Conservative Schemes for Coupled Nonlinear Schrödinger Equations. Numerical Methods for Partial Differential Eq- 
uations, 31, 1814-1843. http://dx.doi.org/10.1002/num.21969

[13] Bhatt, H.P., Abdul, Q. and Khaliq, M. (2014) Higher Order Exponential Time Differencing Scheme for System of Coupled Nonlinear Schrödinger Equations. Applied Mathematics and Computation, 228, 271-291. http://dx.doi.org/10.1016/j.amc.2013.11.089

[14] Xu, Q., Song, S. and Chen, Y. (2014) A Semi-Explicit Multi-Symplectic for a 3-Coupled Nonlinear Schrödinger Equations. Computer Physics Communication, 185, 1255-1264. http://dx.doi.org/10.1016/j.cpc.2013.12.025

[15] Antoine, X., Bao, W. and Besse, C. (2013) Computational Methods for the Dynamics of the Nonlinear Schrödinger/Gross-Pitaevskii Equations. Computer Physics Communications, 184, 2621-2633. http://dx.doi.org/10.1016/j.cpc.2013.07.012

[16] Aydin, A. and Karasozen, B. (2007) Symplectic and Multisymplectic Methods for Coupled Nonlinear Schrödinger Equations with Periodic Solutions. Computer Physics Communications, 177, 566-583. http://dx.doi.org/10.1016/j.cpc.2007.05.010

[17] Bao, W., Tang, Q. and Xu, Z. (2013) Numerical Methods and Comparison for Computing Dark and Bright Solitons in the Nonlinear Schrödinger Equation. Journal of Computational Physics, 235, 423-445. http://dx.doi.org/10.1016/j.jcp.2012.10.054

[18] Chen, A., Zhu, H. and Song, S. (2010) Multi-Symplectic Splitting Method for the Coupled Nonlinear Schrödinger Equation. Computer Physics Communications, 181, 1231-1241. http://dx.doi.org/10.1016/j.cpc.2010.03.009

[19] Ma, Y., Kong, L. and Cao, Y. (2011) High Order Compact Splitting Multi-Symplectic Method for the Coupled Nonliner Schrödinger Equations. Computers and Mathematics with Applications, 61, 319-333. http://dx.doi.org/10.1016/j.camwa.2010.11.007

[20] Talleei, A. and Dehghan, M. (2014) Time-Splitting Pseudo-Spectral Domain Decomposition Method for the Soliton Solutionts of the One-and Multidimensional Nonlinear Schrödinger Equation. Computer Physics Communications, 185, 1515-1528. http://dx.doi.org/10.1016/j.cpc.2014.01.013

[21] Wang, S. and Zhang, L. (2011) Split-Step Orthogonal Spline Collocation Methods for Nonlinear Schrödinger Equations in One, Two, and Three Dimensions. Applied Mathematics and Computation, 218, 1903-1916. http://dx.doi.org/10.1016/j.amc.2011.07.002

[22] Wang, T., Guo, B. and Zhang, L. (2010) New Conservative Difference Schemes for a Coupled Nonlinear Schrödinger System. Applied Mathematics and Computation, 217, 1604-1619. http://dx.doi.org/10.1016/j.amc.2009.07.040

[23] Dehghan, M. and Taleei, A. (2010) A Compact Split Step Finite Difference Method for Solving the Nonlinear Schrödinger Equations with Constant and Variable Coefficients. Computer Physics Communications, 181, 43-51.

http://dx.doi.org/10.1016/j.cpc.2009.08.015 
Submit or recommend next manuscript to SCIRP and we will provide best service for you:

Accepting pre-submission inquiries through Email, Facebook, LinkedIn, Twitter, etc. A wide selection of journals (inclusive of 9 subjects, more than 200 journals)

Providing 24-hour high-quality service

User-friendly online submission system

Fair and swift peer-review system

Efficient typesetting and proofreading procedure

Display of the result of downloads and visits, as well as the number of cited articles

Maximum dissemination of your research work

Submit your manuscript at: http://papersubmission.scirp.org/

Or contact am@scirp.org 